Apidologie, 1977, 8 (4), 451-457.

\title{
ÜBER DIE AUSWIRKUNG VON UMWELTEINFLÜSSEN AUF DIE POPULATIONS - ENTWICKLUNG DER PHYSOKERMES - ARTEN
}

\author{
Action des facteurs du milieu sur le développement \\ des populations des espèces de Physokermes
}

Hermann PECHHACKER

Bundeslehr- und Versuchsanstalt für Bienenkunde Wien Aussenstelle Lunz A-3293 Lunz am See (Österreich)

\section{SUMMARY}

ABOUT THE INFLUENGE OF ENVIRONMENTAL FACTORS

on the POPUlation dynamics of Lecanitdae (Physokermes- spP.)

During 1975 and 1976 investigations began about the influence of different environmental factors on the population dynamics of Lecaniidae. There could not be found an important influence of weather conditions (see PECHHACKER 1976). The temperature sensitivity of $L_{1}$ and the eggs of Lecaniidae seems to be rather small. Some larvae of the first stage surlive up to 11 days at low temperatures as $-13^{\circ} \mathrm{C}$. Even after 12 days exposition to $-13{ }^{\circ} \mathrm{C}$ a part of the eggs (less than $1 \%$ ) could hatch.

In areas with ants (Formica spp.) the number of infestation $\left(L_{2}\right.$, table 2$)$ and survival of overwintering larvae $\left(\mathrm{L}_{2}, \%\right.$, see table 3$)$ was rather greater than in areas without ants. On the other hand the rate of parasitation of adults (in \%, see table 1 , the checking had been done in July/August) was smaller in areas with ants.

So far we see the effect of a real protection on the Lecaniidae due to the ants.

\section{ZUSAMMENFASSUNG}

1975 und 1976 wurde begonnen, die Auswirkungen verschiedener Umwelteinflüsse auf die Populationsentwicklung der Lecanien zu untersuchen.

Es konnte kein wesentlicher Einfluß der Witterung festgestellt werden (siehe PechHacker 1976). Die Temperaturemp findlichkeit der Erstlarven $\left(L_{1}\right)$ und der Eier der Lecanien ist sehr gering. Die $\mathrm{L}_{1}$ überlebten zum Teil bis über 11 Tage bei $-13{ }^{\circ} \mathrm{C}$. Selbst nach 12 Tagen Einwirkung von $-13^{\circ} \mathrm{C}$ schlüpften noch Eier (weniger als $1 \%$ ). 
In Ameisengebieten war die Besatzdichte an Überwinterungslarven $\left(\mathrm{L}_{2}, \mathrm{Tab}\right.$. 2$)$ und die Überlebensrate der im Winter ausgezählten $\mathbf{L}_{2}$ (in $\%$, Tab. 3) deutlich höher, der Parasitierungsgrad der diesjährigen Lecanien (in \%, Tab. 1, im Juli/August ausgezählt) dagegen deutlich geringer. Die Ameisen üben also auch auf die Lecanien eine starke Schutzfunktion aus.

\section{EINLEITUNG}

Durch die große Bedeutung der Physokermes hemicryphus Dalm. sind alle Faktoren bienenwirtschaftlich interessant, welche die Populationsentwicklung der Physokermes-Arten beein flussen.

HöLzL (1956) versuchte z.B. die Populationsschwankungen der Lecanien durch verschiedene Witterungseinflüsse zu erklären. Haragsim (1969) untersuchte den Einfluß der Parasiten und Räuber auf die Populationsentwicklung der Lecanien. Schuutterer (1965) gibt einen ausführlichen Ủberblick über die Bedeutung abiotischer und biotischer Umweltfaktoren auf die Entwicklung der Physokermes-Arten. Fossel (1972) stellte u.a. in Ameisennähe um ein Vielfaches mehr Lecanien fest als in Beständen ohne Ameisen. Wir untersuchten den Einfluß des Windes (Ресннаске r 1971) und verschiedener anderer Witterungsfaktoren auf die Besatzdichte der Lecanien bzw. auf den Honigertrag pro Bienenvolk und Jahr aus der Lecanien-Tracht (Ресннаскев 1976).

Aber alle diese Arbeiten geben noch zu wenig Auskunft darüber, warum in einem Jahr sehr viele Lecanien und schon im nächsten Jahr auf dem gleichen Platz oft nur mehr wenige zu finden sind, bzw. warum oft auf engem Raum starke Unterschiede in der Besatzdichte festzustellen sind.

Die vorliegende Arbeit soll einen zusammenfassenden Überblick über die begonnenen Untersuchungen und über deren erste Ergebnisse geben.

\section{METHODE}

Von 1968 bis 1974 zählten wir auf 4 verschiedenen Plätzen im Winter jeweils die Besatzdichte der Überwinterungslarven $\left(\mathrm{L}_{2}\right)$ aus bzw. wir stellten Anfang August die Zahl der auf geleimten Glasplatten gefangenen Erstlarven $\left(\mathrm{L}_{1}\right)$ pro $\mathrm{m}^{2}$ fest, um zu einer Vorhersage der Fichtentracht für die nächste Periode zu gelangen (Pecheacker 1971 und 1976).

Wir verglichen die Anzahl der dabei gefundenen $\mathrm{L}_{2}$ bzw. $\mathrm{L}_{1}$ (vom August des Vorjahres) und auch die Honigerträge aus der Lecanientracht mit dem Niederschlag und der Monatsdurchschnittstemperatur vom Juni des laufenden und vom August des vorhergehenden Jahres.

1976 wurden zufällige Beobachtungen über die Temperaturempfindlichkeit der $L_{1}$ und der Eier der Kleinen Lecanie gemacht : Es wurden die zur Auszählung des Parasitierungsgrades gesammelten Hüllen aus arbeitstechnischen Gründen am 6.8. in einen Tiefkühlschrank bei $-13{ }^{\circ} \mathrm{C}$ gegeben. Am 12. und 17.8. wurden die $\mathrm{L}_{1}$ und vom 18. - 23.8. noch ca 2000 Eier bei Zimmertemperatur beobachtet.

Die Überwinterungslarven wurden von Ameisengebieten und von Gebieten ohne Ameisen ausgezählt.

1975 und 1976 wurde jeweils im Juli und Anfang August der Parasitierungsgrad der Kleinen Lecanie in einem Gebiet mit Ameisen und in einem Gebiet ohne Ameisen festgestellt. Diese beiden Gebiete lagen ca $12 \mathrm{~km}$ Luftlinie voneinander getrennt im niederösterreichischen 
Waldviertel. In beiden Gebieten herrschten gleiche klimatische und geologische Voraussetzungen. Es wurden nur Hüllen von Lecanien aus dem vorjährigen Maitriebanzatz aus rezählt, um nur Brutblasen von Lecanien aus dem laufenden Jahr nach Parasiten und Räutern zu untersuchen.

Auf acht verschiedenen Plätzen wurden von 200 numerierten Fichten im Winter 1976 (Januar bis März) die $L_{z}$ ausgezählt. Ende Juni wurden dann von den gleichen Fichten die fertigen (mit Eiern oder Parasiten bzw. Räuber) oder noch honigenden Imagines ausgezählt (Methode siehe Pechhacker 1976).

\section{ERGEBNISSE}

\section{Witterungseinflüsse}

In früheren Arbeiten haben wir folgendes festgestellt : Es konnte kein wesentlicher Zusammenhang zwischen $L_{2}$-Besatz pro Quirl und Niederschlag und Durchschnittstemperatur vom August des Vorjahres und vom Juni des laufenden Jahres gefunden werden. Das gleiche gilt für die gefangenen $\mathrm{L}_{1}$ pro $\mathrm{m}^{2}$. Auch der Honigertrag aus der Lecanientracht wird von der Niederschlagsmenge zur Trachtzeit (Juni) nicht beeinflußt. Die Niederschlagsverteilung dagegen hat einen starken Einfluß auf den Honigertrag. Das kann soweit führen, daß die Tracht durch andauerndes Schlechtwetter nich: ausgenutzt werden kann, oder daß sie, wie 1976, durch andauerndes Schönwetter zusätzlich verlängert wird. Hier war auf einem Platz in $300 \mathrm{~m}$ Seehöhe die Honigtauproduktion der Lecanien zwischen 15. und 20. Juni abgeschlossen. Die nicht parasitierten Tiere waren tot und hatten ausschließlich Eier in den Brutblasen. Durch das extrem trockene Wetter wurde aber der auf den Lecanien anhaftende Honigtau nicht abgewaschen. Die Bienen beflogen noch bis 10 Juli Honigtau von der Kleinen Lecanie!

\section{Temperaturemp findlichkeit von $\mathrm{E} i$ und $L_{1}$}

Von den am 6.8.1976 in der mütterlichen Hülle bei - $13{ }^{\circ} \mathrm{C}$ im Tiefkühlschrank aufbewahrten $L_{1}$ waren am 12.8. (nach 6 Tagen) noch mehr als $50 \%$ am Leben. Am 17.8. (nach 11 Tagen) konnten immer noch lebende $\mathrm{L}_{1}$ gefunden werden (weniger als $10 \%$ ). Zwischen 18. und 23.8. schlüpften noch einige Eier (weniger als $1 \%$ ), die ab 18.8. bei Zimmertemperatur gehalten wurden (diese Eier waren ebenfalls vorher 12 Tage bei $-13^{\circ}$ aufbewahrt worden).

\section{Parasitierungsgrad}

Die Zählergebnisse des Parasitierungsgrades sind in der Tabelle 1 dargestellt. Das Jahr 1975 war ein Jahr mit sehr wenig Lachniden. Die Ameisen kümmerten sich außergewöhnlich stark um die Kleinen Lecanien. 1976 dagegen war ein sehr gutes Lachnidenjahr, die Ameisen besuchten überhaupt keine Kleinen Lecanien. Es herrschte im Gegensatz zu. 1975 sehr trockenes Wetter. 
TAB. 1. - Parasitierte oder beraubte Kleine Lecanien in \% (in Klammer die Zahl der jeweils gezählten Tiere).

TaBl. 1. - Pourcentage des petites lécanies victimes de parasitisme ou de prédation (entre parenthèses : nombre des insectes comptés).

\begin{tabular}{c|c|c}
\hline & $\begin{array}{c}\text { Ameisengebiet } \\
\text { Région à fourmis }\end{array}$ & $\begin{array}{c}\text { Gebiet ohne Ameisen } \\
\text { Région sans fourmis }\end{array}$ \\
\hline 1975 & $31,7 \%(429)$ & $67,6 \%(250)$ \\
\hdashline 1976 & $48,5 \%(1250)$ & $68,0 \%(303)$ \\
\hline $\begin{array}{c}\text { Gesamt } \\
\text { Total }\end{array}$ & $44,2 \%(1679)$ & $67,8 \%(553)$ \\
\hline \hline
\end{tabular}

\section{$L_{2}-$ Besatz pro Quirl}

In der Tabelle 2 sind die Besatzdichten an $\mathrm{L}_{2}$ pro Quirl der Ameisengebiete und der Gebiete ohne Ameisen gegenübergestellt. Auf allen Plätzen war 1977 gegenüber 1976 ein starkes Ansteigen der Besatzdichte feststellbar.

TAв. 2. - Besatzdichte von 1976 und 1977 an $L_{2}$ pro Quir (in Klammer die Zahl der insgesamt gezählten Tiere).

TaBL. 2. - Densité de pevplement en $L_{2}$ par verticille on 1976 ot 1977 (entre parenthèses le nombre total d'insectes comptés).

\begin{tabular}{l|c|c}
\hline \hline & $\begin{array}{c}\text { gefundene } \mathrm{L}_{2} \text { pro Quirl (Zahl der } \mathrm{L}_{2} \text { gesamt) } \\
\left.\mathrm{L}_{2} \text { trouvés par verticille (Nombre total de } \mathrm{L}_{2}\right)\end{array}$ \\
\hline $\begin{array}{l}\text { Ameisengebiet } \\
\text { Région à fourmis }\end{array}$ & $0,37(463)$ & 1976 \\
\hline $\begin{array}{l}\text { Ohne Ameisen } \\
\text { Région sans fourmis }\end{array}$ & $0,11(757)$ & $1,30(1590)$ \\
\hline
\end{tabular}

\section{Überlebensrate}

In der Tabelle 3 sind die Ergebnisse der jeweils von den gleichen Fichten ausgezählten $\mathrm{L}_{2}$ bzw. Imagines dargestellt. Es werden Gebiete mit Ameisen und ohne Ameisen auseinandergehalten. 
TAB. 3. - Überlebensrate der im Spätwinter ausgezählten $L_{2}$ (Ergebnisse nur aus dem Jahr 1976).

TABL. 3. - Taux de survie des $L_{2}$ dénombrés à la fin de l'hiver (résultats ne portant que sur 1976).

\begin{tabular}{|c|c|c|c|}
\hline $\begin{array}{c}\text { Gebiet } \\
\text { (Zahl der Fichten, } \\
\text { von jeder Fichte } \\
25 \text { Quirl untersucht) } \\
\text { Région } \\
\text { (Nombre d'épicéas; } \\
\text { pour chacun 25 verticilles examinés) }\end{array}$ & $\begin{array}{l}\text { Im Spätwinter } \\
\text { gezählte } \mathrm{L}_{2} \\
\mathrm{~L}_{2} \text { dénombrés } \\
\text { à la fin de l'hiver }\end{array}$ & $\begin{array}{c}\text { Ende Juni } \\
\text { gezählte Imagines } \\
\text { Imagos dénombrés } \\
\text { à la fin juin }\end{array}$ & $\begin{array}{c}\% \text { der überlebenden } \\
\text { Imagines } \\
\% \text { des imagos } \\
\text { survivants }\end{array}$ \\
\hline $\begin{array}{l}\text { Ameisengebiet (54 Fichten) } \ldots \ldots \\
\text { Region à fourmis (54 épicéas) }\end{array}$ & 462 & 401 & 86,8 \\
\hline $\begin{array}{l}\text { Ohne Ameisen (153 Fichten) } \ldots \ldots \\
\text { Région sans fourmis (153 épicéas) }\end{array}$ & $6 \mathrm{I} 4$ & 405 & 66,0 \\
\hline $\begin{array}{l}\text { Gesamt (207 Fichten) } \ldots \ldots \ldots \ldots \\
\text { Total (207 épicéas) }\end{array}$ & 1076 & 806 & 74,9 \\
\hline
\end{tabular}

\section{DISK USSION}

Es zeigte sich, daß die Witterung nach den bisherigen Erkenntnissen (mit Ausnahme des Windes) offenbar wenig Einfluß auf die Populationsentwicklung der Lecanien hat. Der Wind ist sicher ein Mortalitätsfaktor, aber auch ein Ausbreitungsfaktor (Schmutterer 1965, Pechracker 1971). Es ist aber schwer abzuschätzen, welcher Einfluß überwiegt. Schмutrerer (1965) gibt unter anderem an, daß das $\mathrm{L}_{\mathbf{1}}$-Stadium das empfindlichste von allen Lecanien-Stadien ist (vor allem gegen Niederschlag und niedere Temperaturen). Wir fanden, daß z.B. schon hohe relative Luftfeuchtigkeit die $\mathrm{L}_{1}$ beim Schlüpfen aus der Brutblase hindern kann (Pechнacker 1971). Umso erstaunlicher war es für uns, daß die $L_{1}$ bei so tiefer Temperatur $\left(-13^{\circ} \mathrm{C}\right)$ überleben und daß (wenn auch nur ein ganz geringer \%-Satz) Eier nach einer so langen Kälteeinwirkung noch schlüpfen, obwohl das $L_{1^{-}}$und Eistadium der Lecanie ausgesprochene Sommerformen sind. Die wenigen Ergebnisse über Feststellung des Parasitierungsgrades (nur von 2 Jahren) zeigten bereits, daß die Ameisen einen starken Einfluß auf den Parasitierungsgrad ausüben. Vor allem 1975 ist der Parasitierungsgrad im Ameisengebiet deutlich geringer (nur 31,7\%) als im Gebiet ohne Ameisen (67,6\%). Dies könnte auf den regen Besuch der Kleinen Lecanie durch die Ameisen zur Zeit der Honigtauproduktion zurückzuführen sein (siehe Schmutterer 1965). 1976 war der Unterschied im Parasitierungsgrad bei weitem nicht mehr so hoch wie 1975 (Tab. 1). Die Kleinen Lecanien wurden 1976 trotz bester Honigtauproduktion von den Ameisen nicht beachtet. Die Schutzfunktion der Ameisen ist offenbar eindeutig gegeben 
und in den Jahren mit direktem Ameisenbesuch (in Jahren mit wenig Lachniden) noch deutlich höher. An und für sich ist aber der durchschnittliche Parasitierungsgrad von 44,2\% im Ameisengebiet und von 67,8\% im Gebiet ohne Ameisen bei der hohen Vermehrungsrate der Lecanien in diesen beiden Jahren ungewöhnlich gering gewesen.

Die Besatzdichte an Lecanien ist in den von uns kontrollierten Ameisengebieten in den Jahren 1976 und 1977 deutlich höher als in den Gebieten ohne Ameisen.

Auch bei der Feststellung der Überlebensrate ergab sich im ersten Jahr dieser Untersuchungen ein deutlicher Unterschied zwischen den Ameisengebieten $(86,8 \%$ ) und den Gebieten ohne Ameisen (66,0\%, Tab. 3). 1976 wurden von den im Winter ausgezählten $L_{2}$ im Durchschnitt $74,9 \%$ im Sommer als honigende Lecanien = ausgewachsene Imagines gefunden. Diese Zahl scheint ebenfalls sehr hoch zu sein. Einen richtigen Vergleich werden aber erst weitere Untersuchungen in den nächsten Jahren ermöglichen.

Wir fanden bei diesen Untersuchungen in den ersten Jahren, daß das Wetter offenbar keinen wesentlichen Einfluß auf den Populationsverlauf der Lecanien ausübt. Hingegen fördern die Ameisen deutlich die Lecanien. In Ameisengebieten ist die Besatzdichte höher, der Parasitierungsgrad niedriger und die Überlebensrate größer. Es wurden außer den Ameisen keine entscheidenden Einflußfaktoren auf die Populationsentwicklung der Lecanien bis jetzt gefunden. Was aber verursacht letztlich diese in der Praxis beobachteten starken Populationsschwankungen? Sind es noch nicht festgestellte Krankheiten, die vielleicht die $L_{1}$ oder die $L_{2}$ schon im Frühwinter befallen ? Bei den Zählungen der $L_{2}$ wurden auch immer wieder tote $L_{2}$ gefunden. Es konnte aber auch hier kein Einfluß auf die Populationsentwicklung festgestellt werden (Pecheacker 1976).

Wir fanden bei der Auszählung des Parasitierungsgrades auch vereinzelt ganze Brutblasen voll eingetrockneter $\mathrm{L}_{1}$. Diese Brutblasen wurden aber zu den parasitierten Tieren gerechnet.

Es gibt sicher noch viele Faktoren (was ist über Bakterien- und Viruserkrankungen bekannt?), die in dieser kurzen Beobachtungszeit nicht erfaßt werden konnten.

\footnotetext{
Eingegangen im Mai 1977.

Reçu pour publication en mai 1977.
}

\section{RÉSUMÉ}

En 1975 et 1976 on a entamé des recherches sur l'influence des divers facteurs du milieu sur la dynamique des populations de Lecaniidae. On n'a pas pu mettre en évidence d'action importante des conditions météorologiques (voir PЕсHнACKER, 1976). La sensibilité à la tem- 
pérature des larves du premier stade $\left(L_{1}\right)$ et des œufs des Lecaniidae semble plutôt faible. Certaines larves du $1^{\text {cr }}$ stade parviennent à survivre pendant 11 jours à $-13{ }^{\circ} \mathrm{C}$. Même après 12 jours d'exposition à $-13^{\circ} \mathrm{C}$ une partie des oufs (moins de $1 \%$ ) a pu éclore.

Dans les régions à fourmis (Formica spp.) la densité d'infestation ( $\mathrm{L}_{2}$, tabl. 2) et la survie de larves hivernantes $\left(\mathrm{L}_{2}, \%\right.$, voir tabl. 3) avaient tendance à être plus élevées que dans les régions dépourvues de fourmis. Par contre le taux de parasitisme des adultes (en \%, voir tabl. 1 , le décompte a été fait en juillet/août) est plus faible que dans les régions à fourmis. Celles-ci exercent donc une forte action prốectrice sur les lécanies.

\section{LITERATUR}

Fossel A., 1972. - Die Populationsdichte einiger Honigtauerzeuger und ihre Abhängigkeit von der Betreuung durch Ameisen. Waldhygiene, 9, 185-191.

Haragsim O., 1969. - Aphidophagische Insekten als beschränkende Faktoren der Massenwechsel der Honigtauerzeuger des Waldes. XXII. Apimondia Kongre $\beta$, München, 1969, 437.

HolzL F., 1956. - Warum Fehljahre bei Fichte und Tannc? Bienenvater, 77, 394-305.

Pechiacker H., 1971, - Über die Ausbreitung der Larven der Physokermesarten, speziell von Physokermes hemicryphus DaLM. (Kleine Fichtenquirischildlaus oder Kleine Lecanie). Apidologie, 2, 289-301.

Peсhнacker H., 1976. - Zur Vorhersage der Honigtautracht von Physokermes hemicryphus Dalm. (Homoptera, Coccidae) auf der Fichte (Picea cxcelsa). Apidologie, 7 (3), 209236.

SchmuTtener H., 1965. - Zur Ökologie und wirtschaftlichen Bedeutung der PhysokermesArten (Homoptera, Coccidae) an Fichte in Sïddeutschland. Z. ang. Ent., 56, 300-325. 